\title{
DE CHRISTI HUMANA SCIENTIA ET CONSCIENTIA
}

Quaestio de humana scientia et conscientia Christi medium locum tenet in hierarchia veritatum revelatarum. Ex altera enim parte possessio increatae et infinitae scientiae divinae a homine Jesu consequitur necessario eius veram divinitatem; ex altera parte catholica doctrina de redemptione, quae affirmat Christum hoc modo nos redemisse, quod ,sua santissima passione in ligno crucis nobis iustificationem meruit et pro nobis Deo Patri satisfecit" ${ }^{1}$ supponit eum habuisse rationis usum seu talem scientiam rationalem et humanam sine qua neque meritum neque satisfactio possibiles sunt. Quaestio ergo haec non est purae scholasticae acribiae, sed magni et ponderosi momenti theologici, doctrinalis et religiosi. At quaestio de humana conscientia suae divinitatis necessario iungitur: nam mors Christi inde pracipue suam vim haurit, quod erat libere acceptata a vero Deo Filio, qui homo factus est; suaeque divinitatis concius erat. Recta notio humanae conscientiae divinitatis Christi est proinde praerequisita ad recte intelligendum ipsum opus redemptionis.

Theologici scholastici - cum ante Tridentinum tum post Tridentinum - multum de scientia Chrysti disputabant solebantque admittere in illo increatam scientiam divinam et tres species scientiae creatae: visionem beatificam, scientiam infusam et acquisitam seu experimentalem scientiam. Distinguebant insuper in eo scientiam infusam per se et infusam per accidens. Visionem beatificam - alias scientiam beatam - intelligebant proprio et stricto sensu, prout a Benedicto XII definitia erat; seu beatificam illam vocabant visionem intuitivam et facialem, in qua divina essentia - nulla mediante creatura in ratione obiecti visi se habente - sed divina essentia immediate se nude, clare et aperte ostendente animae beatorum divina essentia perfruuntur ${ }^{2}$. Et affirmabant Christum talem beatificam visionem habuisse sine intermissione a primo instanti suae conceptionis in utero matris per totam vitam neque durante passione in cruce hanc visionem ad momentum amisisse. Jesus, vi huius visionis, per totam vitam terrestrem fruebatur beatitudine caelesti: in utero

1 Enchiridion Symbolorum, ed. Denzinger-Schönmetzer, nr 1529. Siglum: DS.

2 DS 1000. 
matris, durante somno simul ac durante passione in cruce. Ne ad momentum quidem erat simplex viator tendens ad suum ultimum finem, sed sine intermissione erat beatus et comprehensor: beatus, quia caelesti gaudio iam praeditus; comprehensor, quia ultimum finem inamissibiliter possidebat - dum ceteri omnes non sunt in terrestri vita nisi viatores.

Deinde communiter attribuebant Christo scientiam infusam. Nomen „,infusa” primario originem scientiae significat, nam illa vocatur infusa, quae non acquiritur per exercitium propriarum facultatum, sed provenit ex dono Dei illuminantis intellectum. Tunc autem est infusa per se, si talis est naturae, ut numquam per exercitium humanarum facultatum acquiri queat. Quod ideo fit, quia obiectum proprium scientiae infusa per se non sunt res materiales sed substantiae immateriales. Scientia infusa per se est ergo propria spiritibus angelicis, qui mundum materialem cognoscunt in speculo suae immaterialis substantiae. Habens in summo gradu scientiam infusam per se - ex sua natura angelicam - Christus est superior omnibus angelis et primatum respectu illorum detinet. Scientia infusa per accidens novit Jesus quaedam, quae re ipsa non didicit, quamquam discere potuisset: testante ex. gr. evangelio Joannis 7, '15 "mirabantur Judaei, quomodo hic litteras scit, cum non didicerit".

Theologorum consensus quoad supradictas species scientiae in Christo terrestri non amplius adest. Nam - ab anno ca 1960 - plures, qui hac de re scripserunt, non attribuunt Christo terrestri scientiam beatam stricte sumptam, prout in bulla Benedictus Deus definita est; quodsi de quadam visione Dei in Christo terrestri loquuntur, ei diversum sensum dant ab illo, in quo traditionaliter intelligebatur. Etenim communiter opinantur immediatam visionem Dei in Christo terrestri non esse idem ac visionem beatificam beatorum in coelo. Nominandi hic sunt: J. Galot, E. Gutwenger, M. Schmaus, E. Schillebeecx, K. Rahner. Opinante M. Schamus haec visio non attingebat limen cognitionis consciae et secundum E. Schillebeeckx erat idem ac autoconscientia hominis Christi. $\mathrm{K}$. Rahner vero dicit visionem immediatam, vi cuius Christus noverat se esse Deum, non fuisse obiectivam, thematisatam cognitionem Dei Trini sed nonobiectivam conscientiam filiationis divinae, quae simpliciter per hoc actuabatur, quod haec unio re ipsa existebat; nam haec conscentia filiationis divinae nihil aliud est quam intrinseca, ontologica huius filiationis praesentia.

In via aliter interpretandi receptam doctrinam de scientia Christi maxime progressisse videtur $\mathrm{H}$. Riedlinger in quaestione disputata Geschichtlichkeit und Vollendung des Wissens Christi (Freiburg-Wien-Basel 1966). In hoc monographico opere methodice postulavit, ut renuntiaremus non solum scholastico schemati specierum scientiae Christi, sed etiam ipsis nominibus scientiae beatae, infusae, acquisitae; hoc ideo, 
quia haec distinctiones neque cum scripturisticis affirmationibus concordat neque cum hodierno statu psychologiae. Postulavit quoque, ut totalitas scientiae Christi - prout haec prostat in S. Scriptura et in traditione Ecclesiae - unico nomine designaretur: „geschichtliche Gotteschau” seu ",historiae subiecta visio Dei”, ,in qua et historicitas et eschatologica gloria adunantur, modo nobis funditus incomprehensibili" ${ }^{3}$.

E supra enumeratis scientiae speciebus sola scientia acquisita est vere humana; visio vero beatifica - supernaturalibus quoad substantiam et scientia infusa per se - ex definitione angelis propria - nequeunt proprie nominari scientiae humanae. At visio beatifica multum afferre potest ad elucidandam naturam humanae conscientiae divinitatis Christi et ideo de illa hic tractandum est. Econtra - nullum datur argumentum theologicum, quod sufficienter fundet thesim Christum in terris habuisse scientiam infusam per se: nam de tali scientia in Christo neque Scriptura neque Ecclesia neque explicite neque implicite quidquam docent. Deinde scholastici ideo illa Christo attribuerunt, ut fundarent eius primatum respectu angelorum: quod argumentum false supponit principem univoce supra subordinatos eminere. Quodsi haec suppositio vera esset, Deus non esset res regum, est homo. Argumentum ergo non est nisi sophisma.

Consequenter exoptandum est, ut caput de Christi scientia infusa per $\mathrm{se}^{\mathrm{k}}$ dispareat e manualibus christologiae.

Sed quaeritur: caput de scientia vere humana - sic dicta acquisita - Jesu Christi tam elaboratum, egetne quadam accomodata renovatione? Et potestne renovari? Utique - videtur - si in contextu problematis eschatologici discutabitur. Factum omnibus notissimum est, evangelium de regno Dei praesentatum esse a Jesu - secundum evangelia synoptica - in eschatologico horizonte hermeneutico sui temporis. Videbat enim in illo eventum temporis novissimi, qui tam propinquus erat, ut quidam ex astantibus „,non gustabunt mortem, donec videant Filium hominis venientem in regno suo" (Matt 16, 28; Marc 8, 39; Luc 9, 27). Secundum Marc 14, 25 instaurabitur mox post mortem ipsius. Adventus Christi in regno suo est ,dies Domini” (2 Th 2, 2), in qua, postquam evangelium praedicatum fuerit in universo mundo, ,veniet finis" mundi (Matt 24, 14).

Primo ponenda est quaestio facti: utrum Jesus ipse his adamussim verbis et notionibus usus sit, quibus evangelistae usi sunt? Quid in eschatologicis evangeliorum locis ipsissimum factum et dictus Jesu, quid vero posterior eiusdem expressio a traditione formata certitudo scientifica quoad has quaestiones non datur et non nisi cum magna probabilitate af-

${ }^{3}$ H. Riedlinger, o. c., p. 153. 
firmari potest horizontem eschatologicum in suo nucleo provenire ab ipso Christo. Aliis verbis, Christus re ipso usus est in sua praedicatione horizonte eschatologico, quem tamen exacte et cum certitudine determinarè nequimus.

Nunc quaerenda est philosophica et theologica interpretatio huius facti. Philosophica desumitur e hodierna hermeneutica philosophica, quam praesertim - in opere Wahrheit und Methode (1965) H. G. Gadamer exposuit. Hermeneutica discurrit de interpretatione - seu de quaerendo et inveniendo - sensu librorum, eventuum, realitatum. Quod iam Aristoteles in An. post. 1, 1 affirmaverat — „omnem disciplinam, mathesim, e praecedenti cognitione oriri" - factum est unum e principiis hermeneuticae, quod sonat: omnis intellectio Verstehen praesupponit praeintellectionem Vorverstehen. Ad praeintellectionem perinte horizontem hermeneuticum seu complexum omnium quaestionum, quas realitas interpretanda nobis ponit, simul ac complexum notionum, quae ad rectam interpretationem in unaquaque situatione hermeneutica necessariae sunt. Horizon hermeneuticus ab educatione et traditione dependet adeo, ut ad recte intelligendum sensum doctrinae traditae necessarium sit horizontem interpretationis transferri in interpretatione in historicum horizontem traditionis; secus interpretatio deformabit sensum traditionis.

Supradictum principium hermeneuticum - intellectionem supponere semper praeintellectionem - videtur esse bene fundatam adquisitionem gnoseologiae et certitudine gaudere. Valet autem de omni genere interpretativae cognitionis humanae.

Supra indicatae notiones novissimi temporis, novissimae diei, finis mundi, diei Domini et aliae, quibus Jesus usus est in proponenda doctrina eschatologica, mutuatae sunt a traditione prophetica Veteris Testamenti et posterioris iudaismi constituuntque horizontem hermeneuticum, in quo et haec tradictiones et Jesus ipse de futuro progressu historiae salutis loquebantur.

Theologica explicatio - rebus sic stantibus - sonat: Jesus - in acquirenda scientia humana cuiuscumque generis - usus est horizonte hermeneutico sui populi et sui temporis, in quo vixit et crevit: corrigendo quae in illo corrigenda erant et supplendo quae in illo deerant. Ipsi discursus eschatologici evangeliorum synopticorum ostendunt nobis Jesum in actu corrigendi horizontem eschatologicum contemporaneorum ponendo essentiam salutis eschatologicae in interiore et spirituali renovatione et sanctificatione hominis. Quae explicatio non solum certa est, sed etiam formaliter implicite revelata. Primo, est certa, quia non est nisi particularis expressio universalis principii hermeneutici, quod supra vidimus certum esse. Secundo, est formaliter implicite revelata, quia - tam- 
quam casus particularis - continetur"in universali affirmatione He 2, 17: „debuit per omnia fratribus similari”. Sermo enim ibi est de identitate conditionis vitae ethico religiosae nostrae et Jesu Christi. Si quoad omnia similis erat, erat etiam similis quantum ad dependentiam ab horizonte hermeneutico; constat autem ad hanc conditionem pertinere etiam horizontem hermeneuticum, quia horizon atheus vitae religioso ethicae magno obstaculo, christianus autem mogno adiutorio est.

Necessaria dependentia scientiae humanae Christi ab horizonte hermeneutico est hic aspectus, in quo eiusdem authentice humana natura adhuc modo evidentiori elucet quam in eiusdem limitatione et progressu. Videtur ergo omnino introducenda in christologiam catholicam. Bene tamen notandum, haec dependentia non erat absoluta sed relativa tantum, quia - ut visum est - Christus re ipsa corrigebat, quae in recepto horizonte hermeneutico corrigenda erant, et supplebat, quae supplenda erant.

Ex hoc statu dependentiae surgit tamen celebris difficultas: Si humana scientia Christi ab horizonte hermeneutico sui temporis dependebat, eratne ab errore immunis? Quodsi re ipsa ab errore immunis erat, quo modo haec ab errore immunitas explicari potest?

Eschatologismo faventes - ex. gr. A. Loysy, A. Schweizer ut notum est - affirmant Christum re ipsa errasse et deceptionem quantum ad hoc passum esse, quod expectavit adhuc ante suam mortem gloriosum adventum regni Dei. Sancti Patres autem vere unanimes sunt in reiciendo quocumque errore in humana scientia Christi etiam quoad res omnino profana.

Quid ergo? Unanimitas Patrum est criterium doctrinae revelatae, si Patres unanimiter affirmant, expresse vel implicite, aliquam doctrinam esse de fide divina; quodsi hoc non affirmant sunt tamen unanimes, eorum consensus - pariter ac consensus theologorum - non dat nisi scientificam, fallibilem, certitudinem theologicam. Nullus Patrum affirmat immunitatem Christi ab errore esse de fide, omnes tamen vident inconciliabilem oppositionem inter dignitatem divinae personae Christi et errorem. Sed quando de oppositione inter diversas propositiones loquimur, in scientia logica iam sumus, non in fide. Remanet proinde conclusio: theologice certum est Christum in sua humana, acquisita scientia numquam erravit, ne quidem in rebus pure profanis, etiamsi minimi momenti sint.

Evangelia exaltant scientiam Christi, quae se extendebat usque ad cognitionem secretorum cordium; ita ut Jesus „,nosset omnes, et ei opus non erat, ut quis testimonium perhiberet de homine: ipsa enim sciebat, quid esset in homine" (Jo 2, 24-25). Tamen: primo tam Luc 2, 25 quam Jo $2,24-25$ loquuntur de cognitione positionum, quas homines adsume- 
bant - sive positivas sive negativas - per respectum ad doctrinam et personam Christi; quae positiones ad ordinem religiosum, non profanum, spectabant. Secundo - evangelistae nusquam explicant, quo genere scientiae hanc notitiam Christus obtinebat. Tertio - ipsum problema inerrantiae Christi quoad res profanas absens est in evangeliis. Remanet concludendum: e S. Scriptura nihil certum tam pro quam contra omnimodam inerrantiam humanae scientiae Christi erui potest.

Neque theologica ratiocinatio certitudinem afferre potest: quia ignoramus exactam quantitatem humanae intelligentiae Christi; quia quantitativus eius index nobis ignotus est. Ideoque omnis analysis humanae scientiae Christi non est nisi magis vel minus longinqua analogia, non cognoscentes exacte magnitudinem intelligentiae et scientiae humanae Christi, non possumus cum certitudine determinare, quid cum illa compatibile, quid vere incompatibile sit.

Ex quibus conclusio methodologica non parvi momenti sequitur: omnimoda inerrantia humanae scientiae Jesu non constat nisi admissa traditione tamquam norma credendi. Qui traditionem reicit vel ab ea totaliter abstrahit, nullum habebit sufficiens argumentum ad attribuendam Christo omnimodam inerrantiam. Principium protestantismi — ,sola scriptura" - ducit ergo necessario ad mutilandam et deformandam doctrinam de Christo. Quod valet non solum de A. Lysy et A. Schweizer, sed etiam de $\mathrm{H}$. Küng. Traditio autem dogmatica, de qua loquimur, idem est ac perenne et vivum magisterium Ecclesiae.

Ad aliam methodologicam conclusionem provocant evangelici textus loquentes de rerum absentium et secretorum cordium cognitione a Christo. Quaerendum nempe est, quo genere scientiae Christus viderit Natanaelem sub ficu et Lazarum primo aegrotantem deinde autem mortuum esse. Responsio: „,Scientia infusa per accidens” nimis vaga est. Nonne possint hi casus explicari melius per naturalem telepathiam seu - ut dici solet - per naturalem cognitionem praetersensorialem, i. e. non utentem sensibus exterioribus? Exsistunt aliqui homines, qui tali cognitione „telepathica” praeter sensoriali gaudent. Possumusne talem facultatem Christo - perfecto homini - denegare? Quaerendum denique est, utrum in narrationibus evangelicis non inveniantur vestigia non-discursivae cognitionis mysticae, seu cognitionis in "lumine divino". Possumusne talem cognitionem denegare Christo impecabili et sanctissimo? Supraindicatis considerationibus peractis minuetur in vita Christi numerus casuum, in quibus uti debebimus apellatione et notione scientiae infusae per accidens. Desideratur ergo a theologis dogmaticis, ut in tractatu de scientia humana Christi locum reservent cognitioni praetersensoriali et mysticae.

Nunc ad humanam constientiam divinitatis Jesu transeundum est. Quaestio haec intime connectitur cum problemate visionis beatificae in 
Christo terrestri. Inde a medio aevo unanimis theologorum consensus attribuebat Christo terrestri a primo instanti suae existentiae in sinu matris usque ad mortem numquam interruptam visionem beatificam. Sed post a. 1960 - sicut in introductione visum est - plures theologi celeberrimi nominis hanc sententiam reliquerunt et visionem Dei in Christo terrestri aliter interpretari coeperunt. Communis autem nota harum novarum interpretationum est visionem Dei in Christo terrestri neque claram et distinctam neque beatificam fuisse; e visione beatifica, de qua scholastici scripserunt, praeter nomen admodum parum remansit.

In praesenti autem consideratione sermo semper erit de visione beatifica stricto sensu sumpta, quem bulla „Benedictus Deus" definivit. Est autem vere visio, quia est „intuitiva et facialis, nulla mediante creatura in ratione obiecti visi se habente”. Est clara et distincta „divina essentia immediate se nude et aperte... ostendente". Proinde „sic videntes eadem divina essentia perfruuntur", sunt beati et habent vitam aeternam ${ }^{4}$.

Humana conscientia divinitatis Christi videtur - primo aspectu omnino, i. e. metaphysice impossibilis sine visione beatifica. Est enim conscientia: quaedam experimentalis et immediata cognitio sui ipsius seu suae personae. Ergo - vi definitionis - conscientia sui ipsius in Christo erit immediata et experimentalis cognitio divinae personae Verbi, quae est unica persona in Christo. Quia autem Deus simplex omnino est, immediata et experimentalis cognitio personae Verbi est necessario immedia et experimentalis cognitio totius Trinitatis alias totius Dei et est vere beatifica. Praeter visionem beatificam nulla alia experimentalis et immediata cognitio personae Verbi est possibilis. Si ergo Christus non haberet visionem beatificam, necessario careret conscientia suae divinitatis. Hoc est potissimum argumentum pro admittenda visione beatifica in Christo terreștri numquam interrupta. Quod cum ita sit, quaerendum erit, uterum ne vera logice valeat.

Etiam Magisterium Ecclesiae favet interruptae visioni beatificae in Christo antepaschali. Nam S. Officium statuit (5 VI 1918) negative respondendum esse ad quaestionem ,Utrum tuto doceri possit propositio: Non constat, fuisse in anima Christi inter homines degentis scientiam, quam habent beati seu comprehensores" ${ }^{5}$. Et Pius XII in encyclica „Mystici Corporis" attribuit Christo ,beatam illam visionem, qua vixdum in Deiparae sinu conceptus, fruebatur" ${ }^{6}$. Quid ergo?

Omnibus mature perpensis statuendum tamen est: NEQUE MAGISTERIUM NEQUE TRADITIO EXCLUDUNT PROBABILITATEM SEN-

\footnotetext{
4 DS $1000 \mathrm{n}$.

5 DS 3645 .

${ }^{6}$ DS 3812.
}

23 - Analecta Cracoviensia 
TENTIAE, CHRISTUM IN VITA TERRESTRI NON ESSE HABITUALITER GAVISUM VISIONE BEATIFICA, PROPRIE SUMPTA, SUBSTANTIAE DEI TRINI.

Et primo, probabilitati huic minime obstat illud decretum S. Officii vi nempe logicae propositionum modalium. Nam S. Officii est vi missionis suae — statuere, quid sit tutum et quid periculosum pro doctrina fidei et morum. Non tutum seu periculosum pro doctrina est, quod in concretis circumstantii faciliter doctrinae revelatae contradicere potest; quod si re ipsa et actu contradicit, non est amplius periculosum sed simpliciter falsum. Fluunt inde duae conclusiones: propositio tuta est probabiliter revelationi consona et vera, propositio vero periculosa non tuta est probabiliter revelationi contraria et falsa. Deinde - quia finis proprius interventionum S. Oficii est indicare, quid sit tutum, quid non tutum, decreta eiusdem - ex hca ratione - non transcendunt probabilitatem theologicam.

Etiam decretum 5 VI 1918 est solum theologicae probabile. Obiectum proprium eiusdem est propositionem, ,non est certum Christum in terris habuisse visionem beatificam" esse non tutam seu periculosam; seu probabiliter revelationi contrariam et falsam; ex quo - per substitutionem definiti pro definitione - habetur: propositionem „Christus in terris habuit visionem beatificam" esse tutam seu probabiliter revelationi consonem et veram. In his compositis propositionibus adest concursus probabilitatis cum certitudine; propositionibus adest concursus probabilitatis cum certitudine; statuunt enim: probabile est - ex natura decreti esse certam visionem beatificam Christi terrestris. „Probabile est esse certum" autem aequivalet sententiae ,est simpliciter probabile", quia „,peiorem semper sequatur conclusio partem". Quia nemo dat quod non habet, decretum istud, citra suam intentionem non valuit assecurare certitudinem visionis beatificae in Christo terrestri.

Attamen ad definitionem ipsius probabilitatis pertinet, quod non excludit sed positive admittit et includit contrariam propositionem esse veram; si hoc excluderet non amplius est probabilis sed iam certa. Ergo etiam decretum S. Oficii, de quo dicitur, quia tantum probabile est, non excludit sed positive includit probabilitatem sententiae Christum in terris non habuisse permanenter visionem beatificam.

Quoad opinionem Pius XII in encyclica „Mystici Corporis" notandum, illum non tractare ibi ex professo de visione beatifica Christi terrestris, sed per transennam tantum illam tangere sicut et multas alias quaestiunculas utpote synthesis quaedam doctrinae catholicae. In encyclicis autem - ipso Pio XII in encyclica „Humani Generis” 16.8.1950. docente - illud tantum theologice certum est, quod ibi et ex professo tractatur et dirimitur: ,si Summi Pontifices in actis suis de re hactenus controversa 
data opera sententiam ferunt, omnibus patet illam rem... quaestionem liberae inter theologos disputationis iam haberi non passe". Evidens est brevissimum possum de scientia beata Christi hanc conditionem non implere.

Si quae traditio hac de re datur, illa non est nisi consensus latinorum theologorum inde a medio aevo perdurans. Qualem vim habet?. Vim traditionis doctrinalis et dogmaticae an tantum traditionis scientificae et theologicae? Si S. Officium vidisset in illo expressionem doctrinalis traditionis dogmaticae, hic consensus fuisset ei criterium cum certitudine indicans sententiam affirmanem in Christo terrestri scientiam beatam esse revelatam et veram, eius autem negationem - revelationi contrariam et falsam. Ergo: decretum S. Officii non tribuit illi consensui nisi vim scientificae traditionis theologicae, quae tantum valet quantum valent argumenta, quibus innititur.

Unanimis consensus Patrum in hac quaestione deest propter simplicem rationem, quia ipsa notio scientiae beatae erat illis adhuc ignota. Apud illos inveniuntur solum opiniones, quae vel posteriori introductioni scientiae beatae in Christo viam parant vel illam excludunt. Virtualiter excludunt eam omnes, qui Christo ignorantiam diei iudicii universalis attribuunt, dum theoria scientiae beatae in Christo postulat eius relativam omniscientiam, extendentem se ad omnem creaturam et ad omnes creatos actus. Inter admittentes hanc ignorantiam inveniuntur: Origenes, In Matthaeum commentariorum series 55 (PG 13, 1686), Gregorius Nazianzenus, Oratio theologica IV 15 (PG 36, 124), Ambrosius, De fide ad Gratianum 2, 11 (PL 16, 604), Cyrillus Alexandrinus, Thesaurus de sancta et consubstantiali Trinitate, Ass. 22 (PG 75, 368). Quae sunt inter maxima Patrum nomina. Concludendum ergo: hoc in puncto opiniones Patrum ambivalentes sunt.

S. Officium declarans negationem certitudinis quoad exsistentiam scientiae beatae in Christo esse periculosam pro doctrina fidei probabiliter apparentem contradictionem inter hanc negationem et affirmationes IV evangelii respiciebat, quod expressis verbis de ea loqui videtur. Et re vera taliter loci Joannis apparent, usque donec dicta Joannea in exegesi biblico theologica strictae interpretationi subicientur.

Considerationes, quae sequentur, erunt biblico-theologicae: biblicae quia sensum Joannis tangent, theologicae autem, quia sensum Joannis ab exegetis stabilitum in horizonte hermeneutico theologico considerabunt, i. e. in horizonte doctrinae catholicae de Christo et de visione beatifica. Et quaestiones, quibus respondendum erit, quaeque hunc horizontem constituunt, ex hac doctrina catholica desumentur. Considerationes biblico-theologicae praecedentur - sit venia auctori - declarationi personali: auctor perlexit pluries, non solum attente sed etiam studiose, mag- 
nos commentarios in Joannem - e.g. M-J. Lagrange et R. Schnackenbúrg - ita ut quosdam passus memoriter sciat. Quod ad excusandam audaciam propriam dictum sit.

Primo statuitur: HISTORICI FACTI EST JOANNEM NULLIBI SIVE EXPLICITE SIVE IMPLICITE VISIONEM DEI HUMANITATI CHRISTI ATTRIBUISSE. Quae conclusio certitudinem historici „facti” habet. Ad hoc, ut evidens fiat, sufficit attente perlegere omnes 9 locos Jo, in quibus de immediata et experimentali cognitione Dei a Jesu sermo est. En eorum elenchus:

1, 18: Deum nemo vidit umquam; unigenitus Filius, qui est in sinu Patris, ipse enarravit.

3, 11: Amen dico tibi, quia quod scimus loquitur, et quod vidimus testamur.

3, 32-32: Qui de coelo est, super omnes est. Et quod vidit et audivit, hoc testatur.

5, 30: Sicut audio, iudico.

5, 19-20: Non potest Filius a se facere quidquam, nisi quod viderit Patrem facientem; quaecumque enim ille fecerit, haec et Filius similiter facit: Pater enim diligit Fillum et omnia demonstrat ei, quae ipse facit.

8, 38: Ego, quod vidi apud Patrem meum, loquor.

8, 26: Qui me misit, verax est: et ego quae audivi ab eo, haec loquor in mundo.

8, 28: Sicut docuit me Pater, haec loquor.

8, 40: Veritatem vobis locutus sum, quam audivi a Patre.

Nunc ad biblico-theologicam interpretationem. Visio beatifica illa scientia creata est, quae Unigenito Filio Dei convenit ratione humanitatis. Illa vera, quae ei convenit ratione divinitatis, increata et divina scientia est. Quia visio beata in humanitate tamquam in subiecto exsistit et eam afficit, humanitati attribui potest et debet. Quodsi Joannes - in uno saltem eorum locorum, in quibus sermo est de immediata et experimentali cognitione Dei a Christo - hanc cognitionem humanitati attribuisset, clarum esset illud de visione beata loqui. De facto autem nullibi in Jo subiectum attributionis est humanitas sed vel Jesus (loquens in contextu) — 3,$11 ; 3,32 ; 5,30 ; 5,32 ; 8,26 ; 8,28 ; 8,38 ; 8,40$ - vel Unigenitus Filius - 1, 18. Seu semper subiectum attributionis est persona Jesu. Sed adhuc aperta et solenda aliunde remanet quaestio, utrum persona Jesu sit hoc subiectum ratione divinitatis an ratione humanitatis. Inde conclidendum: EX JOANNEM NULLUM ARGUMENTUM CERTUM TRAHI POTEST PRO SCIENTIA BEATA IN CHRISTO TERRESTRI.

Neque vocabularium adhibitum responsionem certam fundat, quia prout recte in suis commentariis theologicis in Joannem R. Schnacken- 
burg asserit - locutiones: „loqui Patris ad Filium, monstrare et docere Patris, discere Filii, audire Filii et videre Filii" non habent strictum theologicum sensum, sed sunt quaedam analogiae (metaphoricae) et similitudines ex humanis adiunctis desumptae. E simplici facto quod 1, 18; 3,$11 ; 3,32 ; 5,19 ; 8,38$ sermo est de visione Dei a Jesu non ergo sequitur illic agi de visione beata Christi terrestris. Necessario recurrendum est ad contextum.

Analysis autem contextus evidenter ostendit in Jo 1,$18 ; 6,46 ; 8,38$; visionem Dei a Filio designare eiusdem scientiam divinam, quia haec visio fundatur in esse Filii in sinu Patris, in eius esse in coelo, in eius esse apud Patrem: Filius autem semper est in sinu Patris, in coelo et apud Patrem, quamquam in mundum descenderat, ratione suae divinitatis, non autem humanitatis. Scientifice, theologice certum ergo est in Jo 1, 18; $46 ; 8,38$ - agi de divina scientis et visione, non autem scientia beata humanitatis Christi probabiliter etiam reliqui 6 Luci sub creatis analogii loquntur de divina scientia Jesu: omnes isti loci insistunt in intima et immediata propinquitate Jesu ad Patrem, quae in Filiation seu in divinitate Jesu fundatur. Quam propinquitatem praesertim commentarii R. Schnackenburg in clara luce ponunt.

Contra omnem expectationem pervenimus ad conclusionem: Joannes non solum non docet quidquam de scientia beata Christi terrestris, sed tribus in locis certe, in ceteris autem probabiliter de scientia divina Christi loquitur. Quia Joannes de scientia beata silet, nequit cum fundamento affirmari negationem eiusdem scientiae in Christo terestri contradicere doctrinae Joannis. Aliis vero is: a Joanne datur ,nihil obstat”.

Quid hac de re reliquum N. Test.? Alium aditum ad interiorem vitam Christi aperit He 2, 17-18; 4, 15. Praecursorem habuit in christologici hymno Phili 2, 6 nn: Jesus: „qui cum in forma Dei esset, non rapinam arbitratus est esse se aequalem Deo, sed semetipsum exinanivit formam servi accipiens, in similitudinem hominum factus et habitu inventus ut homo". „Forma Dei, MORPHE THEOY” - secundum theologicos commentarios J. Glinka ${ }^{7}$ - est idem ac divinus modus exsistendi Dei; „MORPHE DOYLOY, forma servi” est consequenter servilis modus exsistendi servi, in obcedientia Christi manifestatus et exinanitionem eius constituens. „In similitudine hominum factus, EN OMOIOMATI ANTHROPON GENOMENOS” - omoioma est idem ac perfecta imago, ,Reproductio", Abbild. Docetur ergo: Christus in suo servili modo exsistendi factus est perfecta imago hominum: ergo etiam eorum servilis modi exsistendi.

7 J. Glinika, Der Philipperbrief, Freiburg 1968, p. 914. Auctor sequitur hic eius exegesim. 
Quousque se extendat haec perfecta similitudo, in Phili 2 non dicitur. Certissime tanem omnia essentialia, sine quibus forma servi, servilis modus exsistendi et vivendi non datur: quae tamen aliunde noscantur oportet. Ex doctrina de regno coelorum novimus beatos regnare cum Christo (Matth 25, 34; Luc 22, 29) in coelo. Proinde non sunt amplius in forma servorum. Servilis modus exsistendi hominum et regalis modus exsistendi beatorum in coelis videntur essentialiter a se distingui et contradictorie opponi ita, ut impossibile videatur, ut quia sit simul viator et beatus comprehensor. Si haec impossibilitas a Phili 2 doceretur, implicite a S. Scriptura negaretur scientia beata in Christo. Nunc autem haec impossibilitas apparet rationi theologicae tamquam valde probabilis. Ideo concludendum est: PHILI 2 NON SOLUM EXCLUDIT NEGATIONEM HABITUALIS SCIENTIAE BEATAE IN CHRISTO TERRESTRI, SED EI FAVET ET EAM PROBABILEM REDDIT.

$\mathrm{He}$ ulterius progreditur, nam habitualem absentiam eiusdem exigit. In theologico horizonte hermeneutico examinanda sunt primo dicta in $\mathrm{He}$ 2, 17-18 et 4, 15, deinde autem He 5. 8. Quaestiones huius horizontis, quibus considerationes sequentes respondere debent, desumentur primo e doctrina de conditionibus nostrae vitae religiosae et moralis in terra dein autem de statu beatitudinis in coelo.

He 2, 17-18: „Debuit per omnia fratribus similari, KATA PANTA TOIS ADELPHOIS OMOITHENAI, ut misericors fieret et fidelis pontifex ad'Deum, ut propitiaret delicta populi. In eo enim in quo passus est ipse et tentatus, potens est eis, qui tentantur auxiliari, EN OI GAR PEPONTHEN AUTOS PEIRASTHEIS, DYNATAI TOIS PEIRADZOMENOIS BOETHESAI".

He 4, 15: „Habemus pontificem” tentatum per omnia pro similitudine absque peccato, PEPEIRASMENON DE KATA PANTA KATH OMOIOTETA CHORIS AMARTIAS".

Attentione dignum est ambos locos loqui de perfecta et totali similitudine inter Christum pontificem et nos; quod plus est illam similitudinem sistere non in identitate naturae humanae, sed in identitate relationis ad tentationes et probationes huius vitae. Vult dicere: respectu tentationum huius vitae - sive veniant ab infirmitate sive ab hominibus sive a satana - conditio Christi erat totaliter identica cum nostra. A priori nullum genus tentationum a Christo excluditur, quasi dentur aliquae tentationes et probationes nobis solummodo reservatae. Christo excludendum est tamen peccatum.

E 1 Cor 13, 18 scimus conditionem nostrae vitae religiosae et moralis essentialiter consistere in fide, spe et caritate. Ex experientia novimus, quam magnas aliquando difficultates et spirituales pugnas superare debeamus, ut fideles maneamus erga exigentias harum virtutum funda- 
mentalium. Ex quo sequitur: SICUT NOS ITA ET CHRISTUS IN TERRIS DUXIT VITAM FIDEI SPEI ET CARITATIS, SI EIUS RELATIO AD TENTATIONES ET DIFFICULTATES HUIUS VITAE EST RE IPSA IDENTICA CUM RELATIONE NOSERA. Quae conclusio dicenda est necessaria et certa.

Consequentiae huius conclusionis per sequentes syllogiamos conditionales in lucem ponentur: Si Christus in sua terrestri vita habitualiter gaudebat visione beatifica, tunc primo: non ducebat vitam fidei neque spei; secundo: vivens in terris non erat simplex viator, sed simul etiam comprehensor et beatus. Si neque vitam fidei neque spei ducebat ea iam erat beatus et comprehensor, tunc necessario conditiones eius vitae in terris non erant amplius totaliter similes conditionibus nostris, sed sub his duobus respectibus erant essentialiter dissimiles. Aliis verbis: affirmatio habitualis scientiae beatae in Christo terrestri necessario ducit ad negationem doctrinae He 2 et 4 . Idem syllogismus in forma negativa, seu modus tollendo tollens, a negatione ultimae conclusionis incipiens: $\mathrm{Si}$ conditiones vitae Christi in terris erat totaliter similes conditionibus nostris, sicut docetur in He 2 et 4, tunc ducebat vitam fidei et spei neque in terris erat beatus sed simplex viator. Si habebat fidem et spem et erat simplex viator, tunc vivens in terris non gaudebat habitualiter visione beatifica. Aliis verbis: doctrina $\mathrm{He} 2$ et $4 \mathrm{necessario}$ ducit ad excludendam habitualem possessionem visionis beatificae a Christo terrestri.

„Filius Dei - legimus in He 5, 8 - didicit ex iis, quae pasus est, obedientiam, EMATHEN APH ON EPATHEN TEN YPAKOEN" C. Spicq in suo commentario scribit ${ }^{8}$ : „Accentus propositionis ponitur super addiscere obedientiam... In diebus carnis suae Christus expertus est dolorem et hoc modo didicit obedine... Accusativus TEN YPYKOEN originalitatem structurae propositionis praebet". In ulteriores tamen explicationes concludit citando s. Thomam, qui loquitur in contextu habitualis scientiae beatae in Christo: „Didicit obedientiam, id est quam grave sit obedire". Per hoc suis propriis, supra citatis explicationibus, quasi omnem vim aufert: nam aliud est discere obedire, discere obedientiam et discere, quam grave sit obedire. Ex quo exemplo patet: admissa habituali visione beatifica in Christo terrestri impossibile est He 5, 8 explicare secundum sensum, quem postulat ipse textus et contextus. Potius ergo dicendum est: Patienter tolerando dolorem, difficultatem et tentationes - praesertim illos, quos passus est in Gethsemani (He 5, 7) Christus exercebat se in obedientia et exercendo se perficiebat in ea, sicut omnis discipulus perficit se in disciplina, quam addiscit.

\footnotetext{
8 Spicq, L'Epitre aux Hebreux, Paris 1953, vol. II, p. 117.
} 
Iterum duo syllogismi conditionales iuvabunt in percipiendo consequentias huius exegeseos: Si Christus in terris scientia beata habitualiter gaudebat, in virtutibus omnino non progrediebatur. Sed - infert modus tollendo tollens - re ipsa secundum He 5, 8 progressus fecit in obedientia; ergo SCIENTIA BEATA IN TERRIS HABITUALITER NON GAUDEBAT. Quae conclusio probabilis tantum est propterea quod sensus He 5, 8 non est omnino clarus et certus.

Omnia superius dicta de $\mathrm{He} 2,4,5$ reassumuntur in una propositione: SCIENTIFICE (THEOLOGIVE) CERTUM EST IN He 2 et 4 - probabiliter etiam in He 5, 8 - CONTINERI FORMALITER IMPLICITE REVELATAM DOCTRINAM CHRISTUM IN TERRIS NON ESSE GAVISUM HABITUALITER SCIENTIA BEATA.

Dicitur autem ,scientifice certum”, quia haec certitudo tantum valet quantum valent argumenta; potius est certitudo psychologica quam gnoseologica; potius in iudiciis problematicis quam assertoricis exprimitur; possibilitatem denique mutationis vel perfectionis, si qua necessaria est, non solum non excludit sed ex natura sua postulat: sive agatur de correctione a Magisterio sive ab ipsa scientia.

Quodsi carentia scientiae beatae in Christo terrestri a S. Scriptura postulatur, signum certum est, dari aliquem alium modum in tuto ponendi conscientiam humanam divinitatis. Imprimis communissimum argumentum, quod ex necessitate videtur hanc scientiam in Christo terrestri exigere, nullam vim probativam habet: est enim sophisma quod vocatur ignorationis elegchi, quia probat aliud, quam quod probandum erat. Christus - dicitur in illo - ad hoc, ut habeat conscientiam se esse Deum, debuit necessario videre suam personalitatem divinam. Sed nulla est possibilis visio personae divinae praeter visionem beatificam. Ergo - concluditur - Christus in terris habitualiter gaudebat visione beata. Error invenitur autem in prima praemissa, quae minus dicit quam necessarium est. Ad cognoscendum se esse Deum Filium non sufficit enim videre Personam divinam Filii - quam beati vident; necessario exigitur videre re ita ad personam pertinere, ut sit unum cum illa - hanc realem unionem, immo unitatem cum Verbo sola humanitas Christi videt et ideo illi soli conscientia divinitatis copetit.

Ad experimentaliter cognoscendam, seu videndam, hanc realem unitatem cum Verbo - scientia beata non requiritur, quia haec realis unio, realis unitas, creata est creatae humanitatis ad increatam divinitatem. Creata cum sit, in perfectione limitata est ideoque a finito intellectu humano non solum apprehendi, sed etiam comprehendi potest. Haec relatio unitatis cum Verbo tres notas habet, quarum vi conscientiam divinitatis Christi omnino in tuto ponit: Primo - soli humanitati Christi convenit tamquam eius privilegium exclusivum, sicut et solus homo Jesus est 
Deus. Secundo - est necessaria consequentia unionis hypostaticae et duratio eius est eodem modo ininterrupta et aeterna sicut duratio unionis hypostaticae. Tertio - est semper praesens in humanitate et intellectu humano Christi tam ontologice quam gnoseologice. Cum sit semper praesens intellectui, semper ab illo cognosci potest. Quarto cum inseparabiliter unita sit cum infinita gratia unionis, ipsa etiam ad ordinem gratiae pertinet et privilegiis ordinis gratiae gaudet: hoc inter alia quod humanitatem Christi ne minime quidem laesit, sed tantum perfecit.

Laesisset autem, si propter illam humanitas esset privata conscientia suae divinae personalitatis. Unde praesentia huius creatas relationis in humanitate conscientiam divinitatis Christi secumfert. Per eius praesentiam elevatur et habilitatur ad cognoscendum exhautive essentiam eiusdem ita, ut sit praesens per modum illuminationis intellectivae. Intellectus hominis Christi intuendo illam simili modo "videt" in ea terminum, ad quem illa refert humanitatem Christi, quam nos dicimus videre in speculo - seu videre largo sensu - omnia, quae in illo reverberantur stricte loquendo non videt hoc modo ipsum Verbum „nulla mediante creatura in ratione obiecti visi". Conscientia divinitatis - in intuitiva cognitione huius relationis fundata - non est itaque beatifica. Quia haec relatio gnoseologice semper in intellectu praesens est, intellectus Christi potest semper illa uti et ita actualem conscientiam divinitatis habere.

Si de actuali usu huius potentiae agitur, res difficilior fit, quia ,a posse ad esse non valet consequentia" unde distinguendum est. Et quidem: THEOLOGICE CERTUM EST OMNEM ACTUM CONSCIENTIAE HUMANAE IN CHRISTO FUISSE SIMUL ACTUM CONSCIENTIAE DIVINAE. Seu: cognoscendo se esse hunc hominem Christus cognoscebat, a. v. videbat in hac relatione se esse unum cum Deo Filio, se esse Deum Filium.

Sicut gratia unionis est donum datum naturae humanae Christi, ita etiam conscientia divinitatis est donum gratiae datum humanae conscientiae Christi. Ideoque nulla datur ratio, quae ex necessitate exigat actualem conscientiam divinitatis etiam in illis temporibus, in quibus Christus non utebatur conscientia humana, e. g. in somno, in utero matris. Quaedam docta ignorantia hoc nobis necessaria est. Ex altera parte tamen analogia cum contemplatione mystica praemonet nos, ne nimis celeriter ad negationem conscientiae divinae in his temporibus transeamus: observatur enim apud mysticos actus contemplationis infusae tunc profundiores esse quando corum sensus - seu naturalis conscientia - sopiti sunt. Hoc modo probabile fit Christum etiam his temporibus - saltem aliquando - conscientiam suae divinitatis habuisse. Nonne semper? Desu scit. 
Corollarium post scriptum. Theologice certum est Christum a primo rationis usu, seu a prio actu obedientiae erga Deum, iam scivisse - saltem implicite - se vivere ad salvandos omnes homines. 